\title{
Fast and Accurate Segmentation of Dental X-Ray Records
}

\author{
Xin Li, Ayman Abaza, Diaa Eldin Nassar, and Hany Ammar \\ Lane Dept. of Computer Science and Electrical Engineering, \\ West Virginia University, Morgantown, WV 26506-6109 \\ \{xinl, ayabaza, dmnassar, ammar\}@csee.wvu.edu
}

\begin{abstract}
Identification of deceased individuals based on dental characteristics is receiving increased attention. Dental radiographic films of an individual are usually composed into a digital image record. In order to achieve high level of automation in postmortem identification, it is necessary to decompose dental image records into their constituent radiographic films, which are in turn segmented to localize dental regions of interest. In this paper we offer an automatic hierarchical treatment to the problem of cropping dental image records into films. Our approach is heavily based on concepts of mathematical morphology and shape analysis. Among the many challenges we face are non-standard assortments of films into records, variability in record digitization as well as randomness of record background both in intensity and texture. We show by experimental evidence that our approach achieves high accuracy and timeliness.
\end{abstract}

\section{Introduction}

Law enforcement agencies have exploited biometrics for decades as key forensic identification tools. Dental features, resist early decay of body tissues as well as withstand severe conditions usually encountered in mass disasters, which make them the best candidates for PM identification [1] [2]. Recent works on developing a research prototype of an automated dental identification system (ADIS) reveal a couple of challenging image segmentation problems 44. First, the digitized dental X-ray record of a person often consists of multiple films, as shown in Fig. 1(a), which we recognize as a global segmentation problem of cropping a composite digitized dental record into its constituent films. Second, each cropped film contains multiple teeth, as shown in Fig.1(b), which we recognize as a local segmentation problem of isolating each tooth in order to facilitate the extraction of features (e.g., crown contour and root contour) for identification use. The latter problem was studied in [5] [6]. Though performing the film cropping task may seem trivial for a human observer, it is desirable to automate this process and to integrate it with the framework ADIS. In this paper, we focus on the global segmentation (cropping) problem of dental X-ray records and seek a solution to achieve a good tradeoff between accuracy and complexity. On one hand, we want segmentation results to be as accurate as possible since inaccuracy in cropping of 
a)

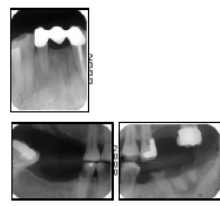

b)

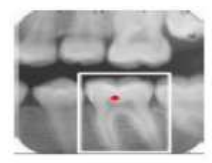

Fig. 1. a) Global segmentation (cropping), b) local segmentation (teeth isolation) [5]

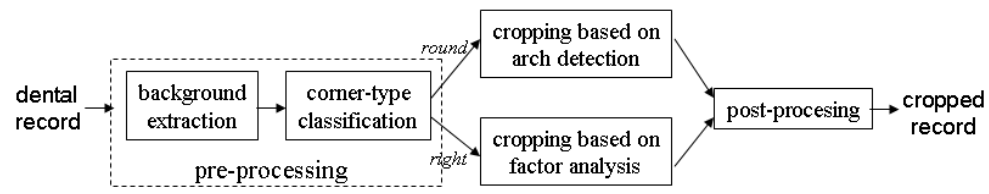

Fig. 2. The three-stages approach for dental record cropping

dental records is likely to hinder the performance of subsequent processing steps and accordingly the overall performance of the entire identification system. On the other hand, we want the computational cost to be reasonably low, especially with the large volume of records that need to be processed. Fast and accurate cropping of dental X-ray records is a nontrivial challenge due to the heterogeneity of dental records. Traditionally, dental X-ray records are digitized by different facilities where human intervention is inevitable during the digitization process. Therefore, characteristics of dental X-ray records vary not only from database to database but also from image to image 3 . We propose a three-stage approach for cropping as depicted in Fig. 2. First a preprocessing stage whereby we extract the background layer of the image record, extract connected components and classify them as either round-corner or right-corner connected components. The second stage performs arch detection and dimension analysis, realization of this stage differs according to the outcome of the preprocessing stage. The third stage is a postprocessing stage that performs topological assessment of the cropping results in order to eliminate spurious objects. In section 2 we introduce the notations and terminology that we will use through the remainder of the paper. In sections [3, 4, and 5] we elaborate on the preprocessing stage, the arch detection and dimension analysis stage, and the postprocessing stage respectively. In section 6 we present experimental results and a discussion on these results. Finally in section 7 , we conclude the paper and sketch our plans for future work.

\section{Notations and Terminology}

In this section, we introduce some notations and definitions for later use. The dental X-ray record is assumed to be a gray-scale image denoted by $X(i, j) \in$ $[0,255]$ where $(i, j) \in \Omega=[1, H] \times[1, W](H, W$ are the height and width of the image). The dimension of individual dental films is denoted by $h, w$. 
a)

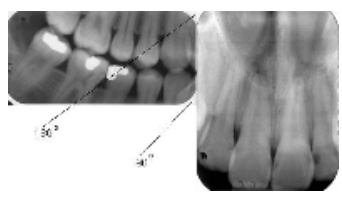

b)

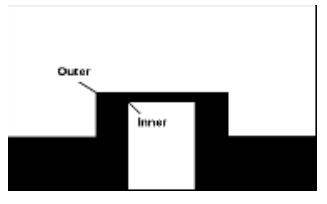

Fig. 3. a) $90^{\circ}$ V-corner and $180^{\circ} \mathrm{V}$-corner; b)Inner L-corner and Outer L-corner

Level set and its size. Level set $L_{k}$ is a binary image defined by $L_{k}(i, j)=$ $\left\{\begin{array}{l}1 X(i, j)=k \\ 0 \quad \text { else }\end{array}\right.$. The size of a binary image $L_{k}$, denoted by $\left|L_{k}\right|$, is simply the total number of ones in the image.

Connected Film Set and Boundary film. Multiple films that are not completely separated form a connected film set. Within a connected film set, a film is called boundary film if removing it does not affect the connectivity of remaining in the set.

Morphological area-open and area-close operators. Area open operator is the extension of morphological opening - it consists of three consecutive filters, namely erosion, small objects removal and dilation. Area close operator is the extension of morphological closing - it consists of three consecutive filters, namely dilation, small objects removal and erosion.

$90^{\circ} \mathbf{V}$-corner and $180^{\circ} \mathbf{V}$-corner. $90^{\circ} \mathrm{V}$-corner refers to the corner formed by a straight line and an arc segment; $180^{\circ} \mathrm{V}$-corner refers to the corner formed by two adjacent arc segments (refer to Fig. 31).

Inner L-corner and Outer L-corner. Inner L-corner refers to a right-corner with one quadrangle of white and three quadrangles of black; outer L-corner refers to a right-corner with one quadrangle of black and three quadrangles of white (refer to Fig. 3).

Note: Inner and outer L-corners can be easily detected by using morphological hit-or-miss operators (as shown in Section 3).

\section{Preprocessing}

\subsection{Background Extraction}

Although the background typically consists of uniform color such as white, gray or black, intensity alone is not sufficient to distinguish the background from dental films. For example, the empty (no tooth) areas in a film often appear as dark regions and could become confused with a black background. Similarly, dental filling often appear as bright regions in a film and could cause problem when the background is white as well. A more robust approach of extracting the background color is to count on geometric clues such as the shape of dental films. Since any dental film can be bounded by a rectangle, the boundary of background largely consists of vertical and horizontal lines. Suppose the histogram of input image $X(i, j)$ and the largest three peaks are $n_{1}, n_{2}, n_{3}$. We consider 
their corresponding level sets $\partial L_{k}, k=n_{1}-n_{3}$ and apply morphological filtering to extract the boundary of those three sets $\partial L_{k}$. For dental films whose boundary is largely rectangular, the fitting ratio of $\partial L_{k}$ by vertical or horizontal lines reflects its likelihood of being the true background. Specifically, we propose to extract vertical and horizontal lines from $\partial L_{k}$ by direct run-length counting and define the fitting ratio by

$$
r_{k}=\frac{\left|R_{k}\right|}{\partial L_{k}}, k=n_{1}-n_{3} .
$$

where $R_{k}$ is the binary image recording the extracted vertical and horizontal lines. The set with the largest fitting ratio among the three level sets is declared to be the background $L_{b}$. As soon as background is detected, we do not need intensity information but only the geometry of $L_{b}$ for further processing (refer to Fig. 4).

(a), (b)

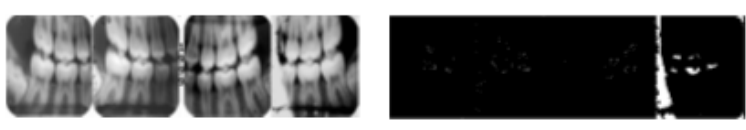

(c), (d)
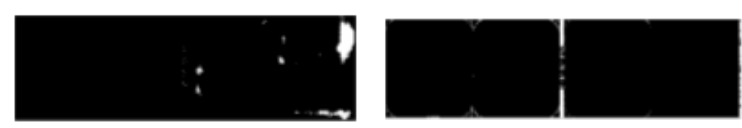

Fig. 4. Background extraction example, a) original dental record, b) level set L1, fitting ratio $\mathrm{r} 1=0.09, \mathrm{c}$ ) level set $\mathrm{L} 2$, fitting ratio $\mathrm{r} 2=0.05$, d) level set $\mathrm{L} 3$, fitting ratio $\mathrm{r} 3=0.36$

\subsection{Arc Detection}

The complement of detected background $\left(\bar{L}_{b}\right)$ consists of non-cropped dental films as well as various noises. The noise could locate in the background (e.g., textual information such as the year) or within dental films (e.g., dental fillings that have similar color to the background). To eliminate those noise, we propose to apply morphological area-open operator to $\bar{L}_{b}$ and $L_{b}$ sequentially. Suppose the Euler number of filtered $\bar{L}_{b}$ is $N$, then we can label the $N$ connected components in $\bar{L}_{b}$ by integers $1-N$. For each connected component (a binary map), we need to classify its corner type since a record could contain the mixture of round-corner and right-corner films. The striking feature of a round-corner film is the arc segments around the four corners. In the continuous space, those arc segments are essentially $90^{\circ}$-turning curves (they link a vertical line to a horizontal one). In the discrete space, we propose to use Hit-or-Miss operator to detect corner pixels first and then morphological area-close operator to locate arc segments. The area-close operator is suitable here because it connects the adjacent corner pixels around a round corner to makes them stand out as an arc segment. By contrast, the corner pixels around a right corner will be suppressed by the area-close operator (refer to Fig. 51). 


\subsection{Dimension Analysis}

For round-corner films, detected arc segments will be shown sufficient for cropping purpose in the next section. However, the location uncertainty of rightcorner film boundary is more difficult to resolve because the inner boundary could disappear in the case of parallel superposition. If all right-corner films are placed in such a way to form a seamless rectangle, it is impossible to separate them apart using geometric information in $L_{b}$ alone. Fortunately, such seamless concatenation does not exist in the database we have, which indicates it is a rare event in practice. Instead, we propose a purely geometric approach for estimating the dimension $(h, w)$ of right-corner films. Our dimension analysis techniques are based on the heuristic observation that concatenation of two right-corner films could only give rise to inner L-corners. Therefore, the distance between any two outer L-corners must be $d_{i}=m_{i} h+n_{i} w, i=1-k_{1}$ where $\left(m_{i}, n_{i}\right)$ are a pair of nonnegative integers. Moreover, since the borders of right-corner films would not all align to each other in the case of non-parallel concatenation, it is reasonable to assume that $\min \left\{d_{i}\right\} \in\{h, w\}$. Referring to Fig. 3 , if we mark the two outer corners corresponding to $\min \left\{d_{i}\right\}$ by $A, B$, then the closest corner to $A, B$ must form a rectangular area of $p h w$ ( $p$ is an unknown positive integer). To further resolve the uncertainty of $p$, we note the constraint on film aspect ratio (i.e., $h \in\left[\frac{2}{3} w, \frac{3}{2} w\right]$ )as supported by an exploratory experiment that we elaborate on in section [6). Such constraint often reduces $p$ to be at most two viable possibilities (combinations of $(h, w)$ ) when $A$ or $B$ is linked to an inner L-corner. If we denote the distance between any two inner L-corners by $d_{i}^{\prime}=m_{i}^{\prime} h+n_{i}^{\prime} w, i=1-k_{2}>k_{1}$, then the weighting coefficients $m_{i}^{\prime}, n_{i}^{\prime}$ could be arbitrary integers. There exist efficient Euclidean algorithm for solving such diophantine equations. By comparing the solutions $\left(m_{i}^{\prime}, n_{i}^{\prime}\right)$ given by different combinations of $(h, w)$, we pick out the one whose solution is closest to integers to be the most likely dimension.

\section{Cropping Techniques}

Preprocessing classifies each connected component in $L_{b}$ to be either roundcorner or right-corner. In this section, we present tailored cropping techniques for round-corner and right-corner components respectively. For round-corner component, we demonstrate that two types of $\mathrm{V}$-corners associated with arc segments are sufficient for cropping. While for right-corner component, we recursively crop out films one-by-one based on the estimated film dimension $(h, w)$.

\subsection{Round-Corner Component}

When multiple round-corner films are placed side by side, they form two types of $\mathrm{V}$-corners as defined above. For $90^{\circ} \mathrm{V}$-corner, its straight edge indicates where the cropping should occur. For $180^{\circ} \mathrm{V}$-corner, we note that it is symmetric with respect to the target cropping line (refer to Fig. 3). Therefore, 
the cropping of round-corner films can be fully based on locating and classifying the two types of V-corners. A V-corner is characterized by the intersection of two segments where the curvature experiences a sharp change. Such geometric singularity can be identified by local analysis of digital arc segment. Specifically, we define "curvature index" at a location $(i, j)$ to be the maximum length of consecutive white pixels in $\bar{L}_{b}$ as we traverse its eight nearest neighbors in a clockwise order. The position is declared to be a V-corner if its curvature index is above 5 and it is close to one of the detected arc segments in corner-type classification. Further classification of V-corner into $90^{\circ}$ and $180^{\circ}$ can be done based on symmetry analysis. For a $90^{\circ} \mathrm{V}$-corner, neither horizontal nor vertical line passing the $\mathrm{V}$-corner divides the corner symmetrically. While for $180^{\circ}$ corner, there exists a symmetric axis which tells the place to cut. We note that unlike generic symmetry detection problems, the direction of symmetric axis is known to be either horizontal or vertical. Therefore, symmetry analysis can be conveniently carried out by correlation or differential techniques.

\subsection{Right-Corner Component}

The cropping of right-corner films is based on the following intuitive observation with the boundary films. Due to the special location of boundary films, they can be properly cropped out with a higher confidence than the rest. Moreover, cropping out boundary films could make other non-boundary films become boundary ones and therefore the whole process of cropping boundary films can be recursively performed until only one film is left. Formally, we propose to characterize the boundary films under a graph theoretic framework. Each film is viewed as a vertex; an edge between two vertexes is induced if the corresponding two films are adjacent to each other. Fig. 6 shows an example of connected film set and its graph representation. It is easy to see that boundary films correspond to the vertexes with degree of one (removing them do not affect the connectivity of the remaining graph). Unless the connected film set forms a loop, it is always possible to reduce the graph by successively removing unit-degree vertexes without affecting its connectivity. To implement recursive cropping, we require reliable detection of boundary films. It follows from the definition of boundary films that they must satisfy: 1) any boundary film contains a pair of outer L-corners; 2) the distance between the L-corner pair is either $h$ or $w$. Therefore, detected outer L-corners in dimension analysis give useful clue for locating boundary films. We note that since the area of connected film set $A_{c f s}$ and the film dimension are both known, the number of films contained in the set it approximately known $\left(n=\left\lfloor\frac{A_{c f s}}{h w}\right\rfloor\right)$. The iteration number of recursive cropping is given by $n-1$.

\section{Postprocessing}

Cropping techniques in the previous section mainly target separating connected films. There are other factors that can not be handled by cropping and may 
a)

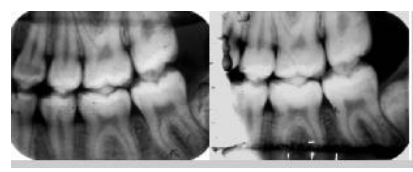

b)

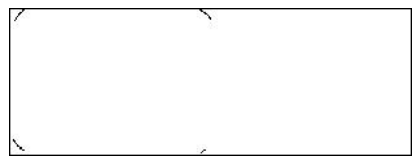

Fig. 5. Arc detection for corner-type classification. a) a dental record with both roundcorner and right-corner films; b) arc detection result.

a)

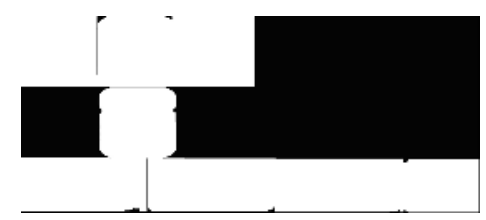

Fig. 6. a)An example of connected film set, b)its graph representation

affect final segmentation results. For example, some dental film is contaminated before digitization such that a portion of film becomes indistinguishable from the background (Fig. 5). The accuracy of cropping itself could also degrade due to errors in dimension analysis. For example, in the case of right-corner films, there might be some leftovers after cropping out $\left\lfloor\frac{A_{c f s}}{h w}\right\rfloor$ films. Consequently, it is desirable to have a post-processing stage to consummate the segmentation results. One of the prior information about dental films is that they are all convex sets, regardless of the corner-type. Such knowledge implies that the hole or cracks of any segmented component be filled in by finding its convex hull. Therefore, the first step in post-processing is to enforce the convexity of all connected components after cropping. Secondly, we propose to check the size and shape of each convex component. If the size of a component is too small or its shape significantly deviates from rectangle, we detect its outer L-corners and check if they correspond to one border of the film. If yes, we conclude that the film was contaminated and derive its boundary using dimension information. Otherwise, we decide it is likely to be a non-film object and put it back to the background layer.

\section{$6 \quad$ Experiments and Results}

In this section we report three types of experiments pertaining to our film cropping approach along with their outcomes: (i) An exploratory experiment to study the constraints on dental films dimensions ratio: Since there are 5 standard film sizes [7, ideally the minimum sides ratio, which we define as the minimum of the aspect ratio and its reciprocal i.e. $\left(\gamma \equiv \min \left[\frac{w}{h}, \frac{h}{w}\right]\right)$, would assume only 5 discrete values $(.5, .64, .66, .75, .77)$. However the manual procedure followed for 
mounting films onto records may result in some variation in the observed values of . In this experiment we used a random sample of 500 manually cropped periapical and bitewing films to study the distribution of $\gamma$. We observed the following: $0.49 \leq \gamma \leq 0.91$ for the entire sample; $\gamma<0.5$ for $\sim 1 \% ; \gamma>0.9$ for $\sim 0.2 \%$; $(0.6 \leq \gamma \leq 0.8)$ in $\sim 94 \%$ of the sample films; and $\sim 6 \%$ of films have $\gamma<0.6$ or $\gamma>0.8$ (almost equally distributed). (ii) A performance assessment experiment: We evaluate both the yield and timeliness aspects of our film cropping approach using a randomly selected test sample of 100 dental records (images) from the CJIS ADIS database [3], the total film count in the test set is 722. We verified that the test sample has variability in background and contains films with both corner types (48 round-corner, and 52 right-corner records). We marked the cropped segments using the following convention: a perfect segment contains exactly one film, an under segmented region contains several whole films 7(b), and an erroneous segment is that contains part of the film, or region from the background texture, or both. In Fig 7(a), we summarize the yield analysis: $\sim 73.7 \%$ of the films were perfectly cropped, $\sim 23.8 \%$ were under segmented, and only $\sim 2.5 \%$ developed into erroneous segments. Further cropping-yield analysis show that in the right corner records perfect segmentation rate is $\sim 70.1 \%$, under segmentation rate is $\sim 28.2 \%$, and erroneous segmentation rate is $\sim 1.7 \%$. While in round corner records these rates are $\sim 76.9 \%, \sim 19.9 \%$, and $\sim 3.2 \%$ respectively. We measured the record cropping time of our algorithm using an uncompiled MATLAB implementation running on a $2.0 \mathrm{GHz} 512 \mathrm{MB}$ RAM Intel Pentium IV PC platform. The average cropping time is $30 \mathrm{kpix} / \mathrm{sec}$ and it varies depending on the number of films in the record and the amount of separation between films. (iii) An exploratory experiment to examine potential future yieldboosting opportunities: Some film geometric properties, like the minimum sides ratio , may provide clues to judge cropped segments. We found that by checking the rule $\gamma>.49$, we could mark under segments, containing $\sim 14.8 \%$ of the films, as $\gamma$-violating. Furthermore, by observing that most records comprise films of about the same area (except for panoramic films), we could also mark under segments, containing $\sim 8.4 \%$ of the films, as area-violating. In the future we may exploit these properties for defining additional postprocessing rules whose violations call for further subsequent processing and hence boost the yield.

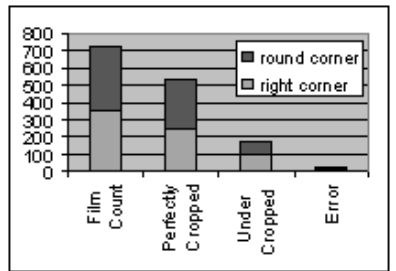

a) b)

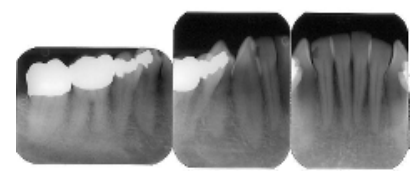

Fig. 7. a)Expiremental results, b)example of undersegmented 


\section{Conclusions and Future Work}

In this paper, we presented a global segmentation technique of cropping the dental films from the dental X-ray records. We started by using the rectangular film property and separating the background that have various colors and textures. Then we classify the connected components according to the corners being right or round. Cutting the round corner components depending on whether they are $90^{\circ}$ or $180^{\circ} \mathrm{V}$-corners; and cutting the right corner ones by viewing the boundary films under a graph theoretic framework, where it is always possible to reduce the graph by successively removing unit vertexes without affecting its connectivity. In the future we will exploit more geometric properties of films to develop additional postprocessing rules, which will identify segments that require further processing by a complementary, more computationally expensive cropping.

\section{References}

1. American Society of Forensic Odontology, Forensic Odontology News, vol (16), No. (2) - Summer 1997

2. The Canadian Dental Association, Communique. May/June 1997.

3. CJIS Division - ADIS, Digitized Radiographic Images (Database), August 2002.

4. G. Fahmy, D. Nassar, E. Haj-Said, H. Chen, O. Nomir, J. Zhou, R. Howell, H. H. Ammar, M. Abdel-Mottaleb and A. K. Jain, "Towards an automated dental identification system (ADIS)", Proc. ICBA (International Conference on Biometric Authentication), pp. 789-796, Hong Kong, July 2004.

5. A. K. Jain and H. Chen, "Matching of Dental X-ray Images for Human Identification", Pattern Recognition, vol. 37, no. 7, pp. 1519-1532, July 2004

6. E. Haj-Said, D. Nassar, G. Fahmy, and H. Ammar, "Dental X-ray Image Segmentation", in Proc. SPIE Biometric Technology for Human Ident., vol. 5404, pp. 409-417, August 2004.

7. S. White, and M. Pharoah, Oral Radiology Principles and Interpretation. Mosby, Inc. Fourth Edition 2000. 